\title{
Risk assessment of dengue fever in Zhongshan, China: a time-series regression tree analysis
}

\author{
K.-K. $\mathrm{LIU}^{1,2,3} \dagger, \mathrm{T} . \mathrm{WANG}^{2,4} \dagger, \mathrm{X} .-\mathrm{D} \cdot \mathrm{HUANG}^{3}$, G.-L. WANG ${ }^{1,2,5}, \mathrm{Y} . \mathrm{XIA}^{1,2}$, \\ Y.-T. ZHANG ${ }^{1,2}$, Q.-L. JING ${ }^{1,6}$, J.-W. HUANG ${ }^{1,2}$, X.-X. LIU ${ }^{2,4}$, J.-H. LU ${ }^{1,2 *}$ \\ AND W.-B. $\mathrm{HU}^{3}$ \\ ${ }^{1}$ School of Public Health, Key Laboratory of Tropical Disease Control of Ministry of Education, Sun Yat-Sen \\ University, Guangzhou, PR China \\ ${ }^{2}$ Zhongshan Research Institute of Public Health, School of Public Health, Sun Yat-Sen University, Zhonghsan, \\ PR China \\ ${ }^{3}$ School of Public Health and Social Work, Institute of Health and Biomedical Innovation, Queensland University \\ of Technology, Brisbane, Australia \\ ${ }^{4}$ Zhongshan Center for Diseases Control and Prevention, Zhongshan, PR China \\ ${ }^{5}$ Haizhu District Center for Diseases Control and Prevention, Guangzhou, PR China \\ ${ }^{6}$ Guangzhou Center for Disease Control and Prevention, Guangzhou, PR China
}

Received 25 November 2015; Final revision 13 September 2016; Accepted 18 October 2016; first published online 22 November 2016

\section{SUMMARY}

Dengue fever (DF) is the most prevalent and rapidly spreading mosquito-borne disease globally. Control of DF is limited by barriers to vector control and integrated management approaches. This study aimed to explore the potential risk factors for autochthonous DF transmission and to estimate the threshold effects of high-order interactions among risk factors. A time-series regression tree model was applied to estimate the hierarchical relationship between reported autochthonous DF cases and the potential risk factors including the timeliness of DF surveillance systems (median time interval between symptom onset date and diagnosis date, MTIOD), mosquito density, imported cases and meteorological factors in Zhongshan, China from 2001 to 2013. We found that MTIOD was the most influential factor in autochthonous DF transmission. Monthly autochthonous DF incidence rate increased by $36 \cdot 02$-fold [relative risk (RR) $36 \cdot 02,95 \%$ confidence interval (CI) $25 \cdot 26-46 \cdot 78$, compared to the average DF incidence rate during the study period] when the 2-month lagged moving average of MTIOD was $>4 \cdot 15$ days and the 3-month lagged moving average of the mean Breteau Index (BI) was $\geqslant 16 \cdot 57$. If the 2-month lagged moving average MTIOD was between $1 \cdot 11$ and $4 \cdot 15$ days and the monthly maximum diurnal temperature range at a lag of 1 month was $<9.6^{\circ} \mathrm{C}$, the monthly mean autochthonous DF incidence rate increased by $14 \cdot 67$-fold (RR 14.67, 95\% CI 8.84-20.51, compared to the average DF incidence rate during the study period). This study demonstrates that the timeliness of DF surveillance systems, mosquito density and diurnal temperature range play critical roles in the autochthonous DF transmission in Zhongshan. Better assessment and prediction of the risk of DF transmission is beneficial for establishing scientific strategies for DF early warning surveillance and control.

Key words: CART model, dengue fever, meteorological factors, mosquito vector, timeliness of diagnosis.

\footnotetext{
* Author for correspondence: Professor J.-H. Lu, School of Public Health, Key Laboratory for Tropical Disease Control of Ministry of Education, Sun Yat-Sen University, Zhongshan 2nd Road, Guangzhou 510000, PR China. (Email: lujiahai@mail.sysu.edu.cn)

$\dagger$ These authors contributed equally to this work.
} 


\section{INTRODUCTION}

Dengue fever (DF), is the most widespread mosquitoborne viral disease globally, with a distribution that includes much of the tropics and subtropics [1]. The increasing geographical expansion and incidence rates of DF constitute a serious global economic, social and public health issue [2, 3]. DF has spread rapidly over the last 50 years as a factor of increased global trade, international travel, climate change, urbanization and limited vector control strategies $[4,5]$. Although the first DF vaccine was licensed in Mexico in December 2015, it will require a lengthy period before it is used worldwide as the effect estimation of the vaccine, where and how to use the vaccine should be considered. As such, DF remains a major public health issue worldwide and a better understanding of the potential risk factors for DF transmission are required to facilitate the development of more effective control strategies and early warning systems.

DF transmission intensity is determined by multifarious risk factors. Many studies focusing on the relationships between DF incidence rates and weather variables are based solely on mean temperature, relative humidity or mean precipitation $[6,7]$. It is, however, still unclear which specific weather elements facilitate the spread of DF across different geographical locations and various climatic regions [8]. Conditions including socioeconomic factors, environmental factors, and human behaviour, could potentially interact with meteorological factors to explain regional differences $[9,10]$. Climatic variation has often been cited as being responsible for differences in the geographical distribution of DF vectors, the reproduction rates of dengue virus (DENV), the extrinsic incubation period (EIP) and mosquito survival [11]. However, findings from recent studies indicate that short-term temperature fluctuations could substantially alter the incubation period for parasites $[12,13]$. Quantitative interpretations of the relationship between meteorological factors and DF incidence rates are scarce.

In 1978, DF re-emerged in Foshan city after a 32year hiatus in China. It subsequently spread widely throughout Guangdong, Guangxi, Hainan Island and Fujian provinces, affecting hundreds of thousands of people in the southeast coastal regions of China [14]. Eighty per cent of reported cases occurred in Guangdong province, including Guangzhou, Zhongshan, Foshan, and the adjacent cities during the past decades. The serotypes of DENV-1-4 have appeared alternately since the outbreak in China in 1978. However, over the past 15 years, DENV-1 has predominated in China [15]. Since the re-emergence of DF, prevention and control have been regarded as a top priority by the Guangdong provincial government. The increasing range of Aedes albopictus has spurred public health concerns as it may further expand the global distribution of DF [16]. While Aedes aegypti is the main vector of DF worldwide, DENV transmission in China is primarily due to $A$. albopictus.

Confirmation of DF cases in China is based on clinical diagnosis and laboratory diagnosis. Early notification of DF cases identified in primary and secondary care is crucial for identifying outbreaks and initiating an early response. Early diagnosis of DF is challenging [17]. The incubation period of patients infected with DENV for transmission via mosquitoes is $4-5$ days (maximum 12 days), and the average time interval between symptom onset and laboratory confirmation of DF patients is 6 days in China [18]. Timely diagnosis and early treatment of patients are essential, especially for severe cases. Furthermore, cases that remain undetected or are not identified in a timely manner represent a potentially important risk factor in the spread of DF virus. Few studies have quantitatively assessed the timeliness of diagnosis of autochthonous DF. The aim of the current study is to identify the hierarchical relationship between timeliness of DF surveillance systems, mosquito density, imported cases, meteorological factors and autochthonous DF incidence rates, and to estimate the threshold effects of highorder interactions among these risk factors on the incidence rates of autochthonous DF. This information will be beneficial in guiding future approaches for the control of DF in Guangdong province and beyond.

\section{MATERIALS AND METHODS}

\section{Study site}

The study site was located in Zhongshan city (latitude $22.515847^{\circ} \mathrm{N}$, longitude $113.392207^{\circ} \mathrm{E}$ ), which is in south-central Guangdong province in the port of the Pearl River Delta. Zhongshan is close to Guangzhou (the capital city of Guangdong), Hong Kong and Macau. In 2012 the population of Zhongshan was 3155078 [19]; there is, however, substantial movement of people between Zhongshan and adjacent cities which 
may facilitate the spread of disease. Zhongshan city has a subtropical monsoonal climate with hot, humid summers and mild, dry sunny winters. The annual mean temperature is between $21^{\circ} \mathrm{C}$ and $23{ }^{\circ} \mathrm{C}$, and the annual total precipitation is $2010 \mathrm{~mm}$ (Fig. 1).

\section{Data collection and case definitions}

Notification of DF is a legally mandated in in China, and cases are diagnosed according to the national diagnostic criteria of DF [20, 21]. Data on both autochthonous and imported cases were obtained from the Notifiable Infectious Disease Reporting System (NIDRS) at Zhongshan Center for Disease Control and Prevention (CDC) for the period 1 January 2001 to 31 December 2013. The DF diagnosis criteria included epidemiological exposure history, clinical manifestations, and laboratory tests. Since DF is a notifiable disease, positive test results need to be reported by hospitals to China CDC. DF was clinically diagnosed according to the following symptoms: presenting fever $39-40{ }^{\circ} \mathrm{C}$ and with one of the following symptoms: rash, headache, retro-orbital pain, myalgia, arthralgia, rash and conjunctival congestion. The serum samples of suspected patients were then sent to the local CDC laboratory for confirmation based on the following different laboratory tools: the detection of the virus isolation and identification, viral nucleic acid positive by PCR test, detection of antigens or antibodies, a fourfold increase in specific IgG antibody titre in the paired serum specimens in the acute and convalescent phase or the combination of these methods [22]. The laboratory-test tools for DF serum are combined on the basis of the days of illness phases. The sensitivity of each approach is influenced by the duration of the patient's course of illness. The ratio $\operatorname{IgM} / \operatorname{IgG}$ is used to identify primary and secondary DENV infections [22]. Autochthonous DF cases were defined as the confirmed cases in a patient with no history of travel to endemic areas outside China or other cities in China in the preceding 14 days. Conversely, at the city level, imported DF cases were defined as the patients with DF who had traveled to dengue-endemic areas (as specified by WHO) outside China or other cities in China in 14 days prior to the development of symptoms [23].

The symptoms of DF are non-specific and may mimic other febrile illnesses. As such, clinical diagnoses lack specificity and laboratory confirmation is generally sought. For every notified DF case, the date of symptom onset and the date of laboratory diagnosis were recorded. The timeliness of diagnosis was defined as the monthly median time interval between symptom onset date and laboratory diagnosis date (MTIOD).

The population statistics data were obtained from Zhongshan Statistical Bureau [19]. Previous studies have reported that meteorological factors such as temperature, rainfall, relative humidity, and diurnal temperature range (DTR) are related to DF incidence rates $[6,13]$. DTR is defined as the difference between the maximum and the minimum daily temperature. In this study, monthly meteorological data were provided by Zhongshan Meteorological Bureau [24]. Meteorological variables used in the study included: monthly maximum temperature (MaxT) $\left({ }^{\circ} \mathrm{C}\right)$, monthly minimum temperature (MinT) $\left({ }^{\circ} \mathrm{C}\right)$, monthly mean temperature (MeanT) $\left({ }^{\circ} \mathrm{C}\right)$, monthly maximum diurnal temperature range (MaxDTR) $\left({ }^{\circ} \mathrm{C}\right)$, monthly mean diurnal temperature range (MeanDTR) $\left({ }^{\circ} \mathrm{C}\right)$, monthly minimum diurnal temperature range (MinDTR) $\left({ }^{\circ} \mathrm{C}\right)$, monthly mean rainfall (MeanRF) (mm), monthly maximum relative humidity (MaxRH) (\%), monthly minimum relative humidity (MinRH) (\%), and monthly mean relative humidity (MeanRH) (\%).

A. albopictus is the sole vector for the transmission of DENV in Zhongshan, China [25]. Mosquito surveillance, especially larval density surveillance is routinely performed in Zhongshan. Larval density surveillance is routinely performed in the 24 administrative districts of Zhongshan. Breteau index (BI) was defined as the number of positive containers containing larvae per 100 houses inspected by monthly door-to-door interview [22]. BI is considered as the most informative index for A. albopictus density due to inclusion of the information of positive containers and houses [22]. The surveillance was conducted at monthly intervals over the study period.

\section{Statistical analysis and modelling}

\section{Time-series cross-correlation analysis}

As the relationship between DF incidence rates and other factors for the current month or previous months require exploration, cross-correlations were used to compute correlations between meteorological factors and the DF incidence over a range of time lags (a time lag was defined as the timespan between the risk factors and incidence rates of DF fever). The cross-correlation function was performed to quantify the linear correlation between the monthly autochthonous DF cases and risk factors, including 


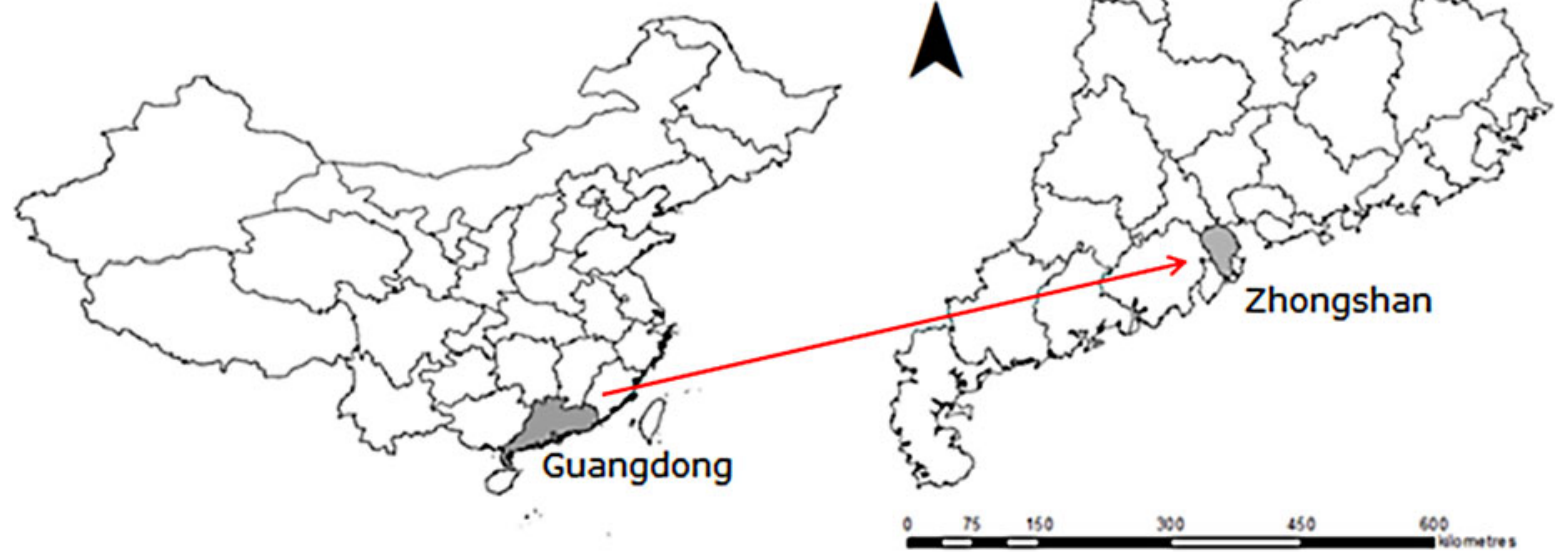

Fig. 1. Location of Zhongshan, Guangdong, China.

the monthly imported cases, meteorological variables, mosquito density and monthly MTIOD over a lag time of 7 months. In this study, we used the lagged moving average (MA) value to calculate the lagged effect on the series of the factors. The significant time lags of the risk factors were chosen for the calculation of lagged MA. The MA value is applied in a time-series model which is defined as the mean value of the previous numbers of lagged periods [26]. The lagged MA could be better for explaining the lagged effects of the factors.

\section{Time-series classification and regression tree}

The classification and regression tree (CART) model is a flexible, robust and non-parametric statistical method which is useful for the analysis of complex ecological applications. Exploring and constructing the decision-tree model in the ensemble method can express the nonlinear relationships, high-order interactions, and missing values.

CART is suitable for predicting the relationships between dependent variables and exploratory variables [27]. It can interpret the variation of a single response variable by recursive partitioning of the the ecological data into more homogeneous subsets, using combinations of explanatory variables. The response variable can be a categorical variable (a classification tree) or a continuous variable (a regression tree) [28]. A time-series regression tree model was used to determine the threshold effects of the risk factors in the monthly incidence rates of autochthonous DF. In this study, we used meteorological variables, mosquito density, timeliness of diagnosis and the seasonality factor [harmonic factors: $\sin (2 \mathrm{pt} / 52)$ and $\cos$ $(2 \mathrm{pt} / 52)]$ as the independent variables for analysis in the CART model. The tenfold cross-validation was performed to identify the hypothesis in the analysis.

The tree was grown by the recursive binary splitting of variables, including meteorological variability, mosquito density, and timeliness of diagnosis. Each split formed on the basis of a single explanatory variable and two nodes, which was selected to maximize homogeneity (minimize impurity) of the resulting two nodes. Homogeneity was assessed with several impurity functions, and two measures were used for regression trees: least squares (Gini splitting rules) and least absolute deviations (two splitting rules). During the process of modelling, a preferred method is to produce an overly large tree and then prune and test using independent data. Pruning is used to reduce the tree to an optimal size, which is determined using cross-validation or independent tests. Cross-validation is used to choose the best tree on the basis of the misclassification error and the complexity of the tree. The task is achieved through cost-complexity function: $R_{C}(T)=R(T)+c(\tilde{T}) \rightarrow \min T$, where $R(T)$ is the residual estimate of error, misclassification error of the tree $T, c(\tilde{T})$ is the complexity measure which depends on $\tilde{T}$, which is the number of terminal nodes in the tree, $c$ is the parameter which is used to build the tree through learning sample and testing sample [29]. The procedure of tenfold cross-validation is performed to prune the overly large trees and to select the best-trimmed tree through a training data subgroup and a testing data subgroup. The 
process of cross-validation is performed as follows: the data are randomly split into ten subsets, training the model on nine subsets and calculating the estimated error rates on the tenth subset. The best optional tree is the one with the smallest estimated error rates [27].

Monthly DF incidence was considered a continuous response variable when the regression model was built in this study. The risk factors which were significantly positively or negatively associated with the autochthonous incidence rates in cross-correlation analyses were applied to the time-series regression tree model as independent variables. All time-series variable graphs were completed by Microsoft Excel 2007 (Microsoft Corp., USA). Cross-correlation between the factors and DF incidence rates were performed using SPSS v. 16 (SPSS Inc., USA) and time-series regression tree models were constructed in R software v. 3.1.1 (R Foundation for Statistical Computing, Austria).

\section{Ethics statement}

Ethics clearance for this project was approved by Sun Yat-Sen University and Zhongshan Center for Disease Control and Prevention Ethical Review Committee (Approval no: 2015024). DF data were collected from NIDRS. All potentially identifiable information has been removed to protect the privacy of individuals.

\section{RESULTS}

\section{Descriptive analysis}

The seasonal variations of autochthonous DF cases, meteorological factors, mosquito density and MTIOD during the study period are shown in Figure 2. There were 1110 notifications for autochthonous DF cases and 40 imported cases in Zhongshan city during the period 1 January 2001 to 31 December 2013. The autochthonous cases mainly occurred between July and November. The mean monthly number of autochthonous cases was $7 \cdot 12$ (Table 1).

\section{Cross-correlation analysis}

The results of the cross-correlation function show that autochthonous DF incidence rates were significantly positively associated with MaxT, MTIOD, and MeanBI at 1-3 months lagged MA, with monthly imported DF cases at a lag of 1 month, with MinT and MeanT at 1-2 months lagged MA, with MaxDTR, MeanRH, MaxRH at a lag of 1 month. MinRH was positively associated with the DF incidence rates at a lag of 2 months (Fig. 3, Supplementary Table S1). There was no significant relationship between MaxRF, MeanRF, MeanDTR, MinDTR and autochthonous DF incidence rates (Supplementary Fig. S1, Supplementary Table S1).

\section{The regression tree model}

We calculated the lagged MA values with the significantly lagged orders based on the cross-correlation analysis. The significant factors from cross-correlation at the different time lags were used for the construction of regression tree models (Fig. 4). For the period 1 January 2001 to 31 December 2013, the regression tree model demonstrated MTIOD was the most important splitting factor contributing to DF incidence rates, with a threshold value of 4.15 days (Fig. 4). The model also showed that mosquito density was of greater importance than the meteorological factors temperature range. For example, the monthly incidence rates of autochthonous DF increased by 26.43-fold [relative risk (RR) 26.43, 95\% confidence interval (CI) $11 \cdot 74-41 \cdot 13$, compared to the average DF incidence rate during the study period] when the 2-month lagged MA of MTIOD was $>4 \cdot 15$ days. In the right branch of the tree, the monthly autochthonous DF incidence rates increased by 36.02 -fold (RR $36 \cdot 02,95 \%$ CI $25 \cdot 26-46 \cdot 78$, compared to the average DF incidence rate during the study period) when the 3 -month lagged MA of MeanBI was $\geqslant 16.57$ and the 1-2 months lagged MA of MTIOD was $>4 \cdot 15$ days. MTIOD and MaxDTR were included in the left branch of the tree. If the 1-2 months lagged MA of MTIOD was between $1 \cdot 11$ and $4 \cdot 15$ days and MaxDTR at a lag of 1 month was $<9 \cdot 6^{\circ} \mathrm{C}$, the monthly mean autochthonous DF incidence rate increased by $14 \cdot 67$-fold (RR 14.67, 95\% CI $8 \cdot 84$ $20 \cdot 51$, compared to the average DF incidence rate during the study period). However, the other branch showed the autochthonous DF incidence rate increased by 3.06-fold compared to the overall monthly mean autochthonous DF incidence rate when the 1-2 months lagged MA of MTIOD was between $1 \cdot 11$ and $4 \cdot 15$ days and MaxDTR at a lag of 1 month was $\geqslant 9 \cdot 6{ }^{\circ} \mathrm{C}$ (RR $3 \cdot 06,95 \%$ CI $1 \cdot 17$ 4.95) (Fig. 4). 

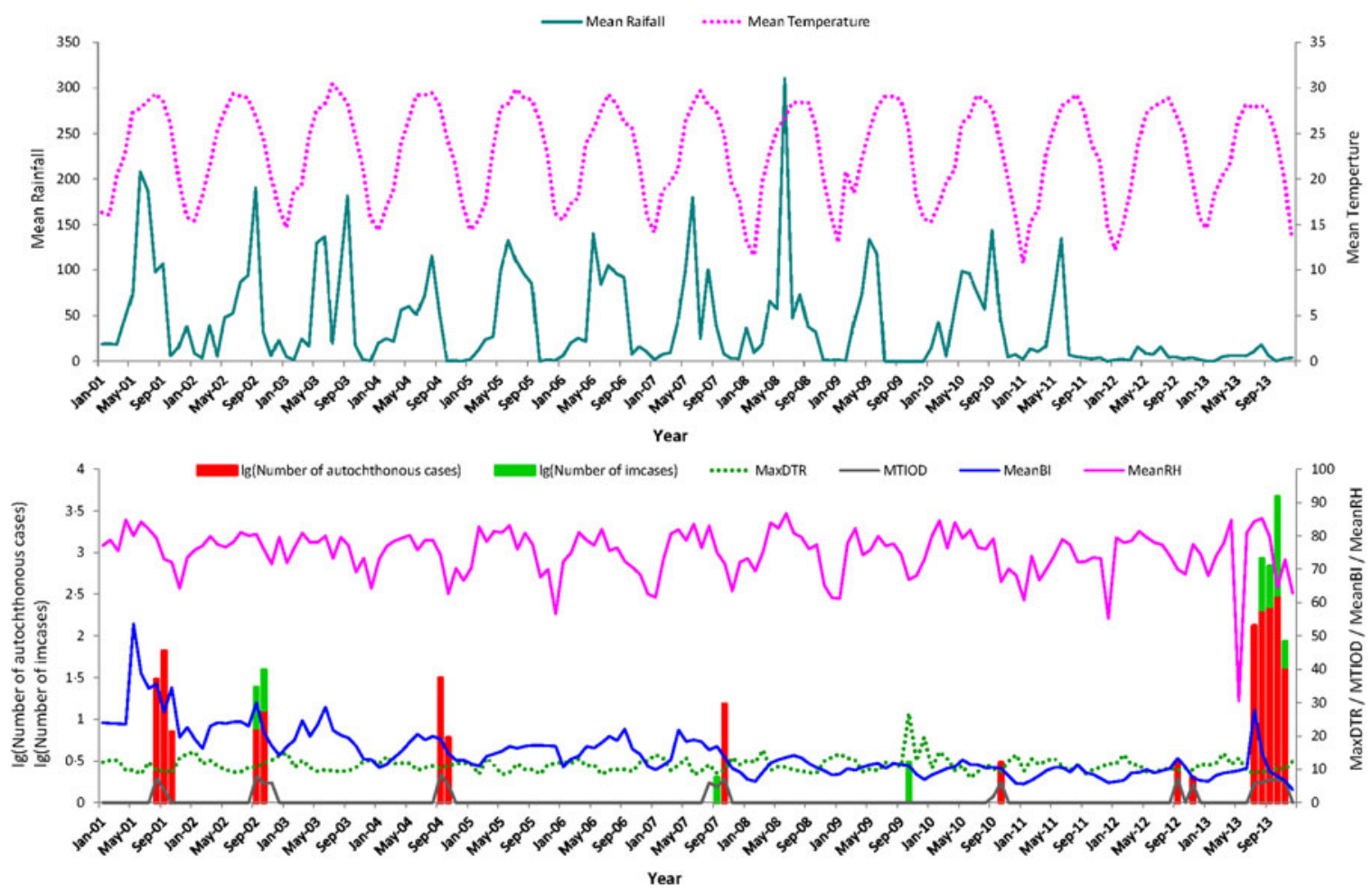

Fig. 2. Monthly distribution of autochthonous dengue cases, imported dengue cases, mosquito density, meteorological variation and time interval between onset and diagnosis from January 2001 to December 2013 in Zhongshan, China. Mean rainfall $(\mathrm{mm})$, monthly mean rainfall; mean temperature $\left({ }^{\circ} \mathrm{C}\right)$, monthly mean temperature; MaxDTR $\left({ }^{\circ} \mathrm{C}\right)$, monthly maximum diurnal temperature range; MTIOD (days), monthly median time interval between symptom onset and diagnosis by day; MeanBI, monthly mean Breteau Index; MinRH (\%), monthly minimum relative humidity.

Table 1. Summary statistics of monthly data for all variables between 1 January 2001 and 31 December 2013 in Zhongshan, China

\begin{tabular}{|c|c|c|c|c|c|c|}
\hline Variable & Mean & ${ }^{P}$ S.E.M. & Minimum & Maximum & 1st quartile & 3rd quartile \\
\hline $\mathrm{ADF}^{\mathrm{a}}$ & $7 \cdot 115$ & $2 \cdot 946$ & 0 & 310 & 0 & 0 \\
\hline $\mathrm{IDF}^{\mathrm{b}}$ & $0 \cdot 26$ & $0 \cdot 108$ & $1 \cdot 343$ & $1 \cdot 805$ & 0 & 0 \\
\hline MeanBI ${ }^{\mathrm{c}}$ & $14 \cdot 39$ & $0 \cdot 6$ & 3.908 & $53 \cdot 586$ & $9 \cdot 59$ & $18 \cdot 179$ \\
\hline MTIOD $^{d}$ & $6 \cdot 044$ & $0 \cdot 337$ & $1 \cdot 833$ & $8 \cdot 384$ & $5 \cdot 5$ & $7 \cdot 045$ \\
\hline MeanT $^{\mathrm{e}}$ & $22 \cdot 907$ & $0 \cdot 425$ & $10 \cdot 745$ & $30 \cdot 41$ & $18 \cdot 344$ & $27 \cdot 889$ \\
\hline $\operatorname{MaxT}^{\mathrm{f}}$ & $31 \cdot 790$ & $0 \cdot 303$ & $19 \cdot 400$ & $38 \cdot 700$ & $28 \cdot 750$ & $32 \cdot 000$ \\
\hline $\operatorname{MinT}^{\mathrm{g}}$ & $15 \cdot 318$ & $0 \cdot 581$ & $1 \cdot 100$ & $26 \cdot 200$ & $8 \cdot 125$ & $16 \cdot 000$ \\
\hline MeanDTR $^{\text {h }}$ & $6 \cdot 801$ & $0 \cdot 065$ & 4.997 & $9 \cdot 248$ & $6 \cdot 288$ & $7 \cdot 219$ \\
\hline MaxDTR $^{\mathrm{i}}$ & $11 \cdot 276$ & $0 \cdot 178$ & $7 \cdot 4$ & $26 \cdot 8$ & $9 \cdot 7$ & $12 \cdot 475$ \\
\hline $\operatorname{MinDTR}^{\mathrm{j}}$ & $2 \cdot 550$ & $0 \cdot 077$ & $0 \cdot 800$ & $5 \cdot 900$ & $1 \cdot 800$ & $3 \cdot 100$ \\
\hline MeanRF $^{\mathrm{k}}$ & $40 \cdot 681$ & $4 \cdot 207$ & 0 & $310 \cdot 467$ & $3 \cdot 99$ & $64 \cdot 194$ \\
\hline $\operatorname{MaxRF}^{1}$ & $404 \cdot 680$ & $40 \cdot 621$ & $0 \cdot 000$ & $3258 \cdot 000$ & $46 \cdot 275$ & $658 \cdot 000$ \\
\hline MeanRH ${ }^{\mathrm{m}}$ & $75 \cdot 036$ & $0 \cdot 569$ & $30 \cdot 417$ & $86 \cdot 733$ & $72 \cdot 105$ & $79 \cdot 637$ \\
\hline $\operatorname{MaxRH}^{\mathrm{n}}$ & $91 \cdot 465$ & $0 \cdot 522$ & $34 \cdot 524$ & $100 \cdot 000$ & $89 \cdot 000$ & $95 \cdot 000$ \\
\hline $\mathrm{MinRH}^{\mathrm{o}}$ & $33 \cdot 042$ & $0 \cdot 816$ & $8 \cdot 000$ & $53 \cdot 000$ & $25 \cdot 663$ & $33 \cdot 000$ \\
\hline
\end{tabular}

${ }^{\mathrm{a} A D F}$, Monthly autochthonous DF cases; ${ }^{\mathrm{b}} \mathrm{IDF}$, monthly imported DF cases; ${ }^{\mathrm{c}} \mathrm{MeanBI}$, monthly mean Breteau Index; ${ }^{\mathrm{d}}$ MTIOD (day), monthly median time interval between symptom onset and diagnosis; ${ }^{\mathrm{e}} \mathrm{MeanT}\left({ }^{\circ} \mathrm{C}\right)$ : monthly mean temperature; ${ }^{\mathrm{f}} \mathrm{MaxT}$, monthly maximum temperature; ${ }^{\mathrm{g}} \mathrm{MinT}\left({ }^{\circ} \mathrm{C}\right)$, monthly minimum temperature; ${ }^{\mathrm{h}} \mathrm{MeanDTR}\left({ }^{\circ} \mathrm{C}\right)$, monthly mean diurnal temperature range; ${ }^{\mathrm{i}}$ MaxDTR, monthly maximum diurnal temperature range; ${ }^{\mathrm{j}} \mathrm{MinDTR}\left({ }^{\circ} \mathrm{C}\right)$, monthly minimum diurnal temperature range; ${ }^{\mathrm{k}}$ MeanRF, monthly mean rainfall; ${ }^{1} \mathrm{MaxRF}(\mathrm{mm})$, monthly maximum rainfall; ${ }^{\mathrm{m}} \mathrm{MeanRH}(\%)$, monthly mean relative humidity; ${ }^{\mathrm{n}} \mathrm{MaxRH}(\%)$, monthly maximum relative humidity; ${ }^{\circ} \mathrm{MinRH}(\%)$, monthly minimum relative humidity; ${ }^{\mathrm{p}}$ S.E.M., standard error of mean. 

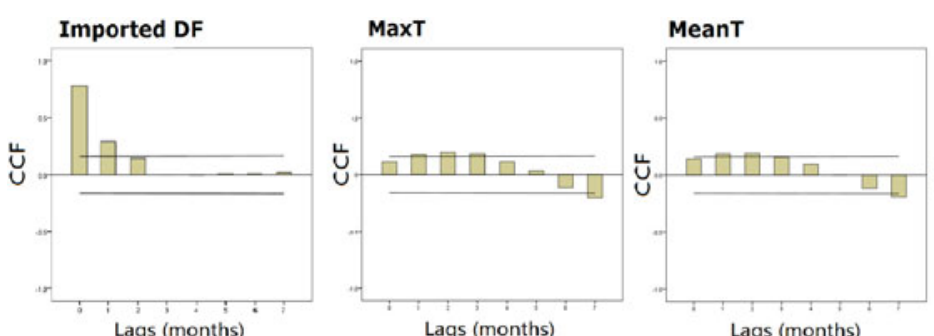

Lags (months)

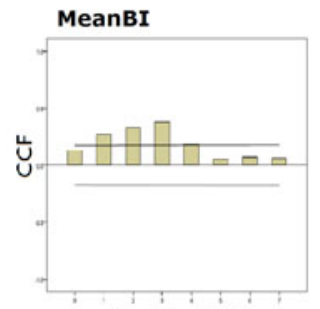

Lags (months)

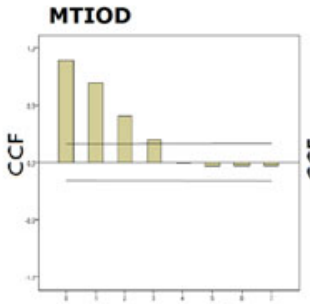

Lags (months)

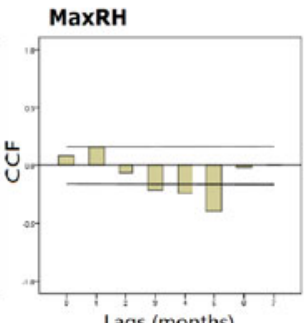

Lags (months)

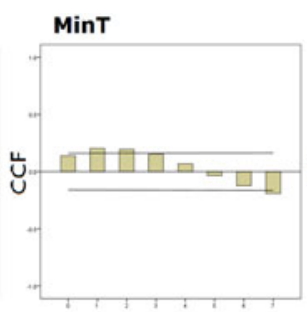

Lags (months)

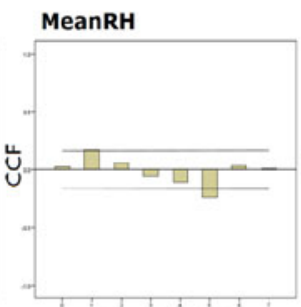

Lags (months)

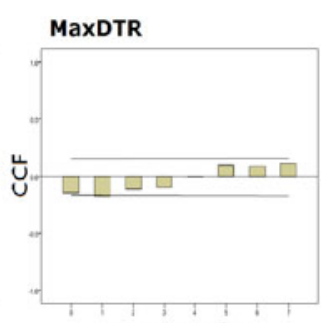

Lags (months)

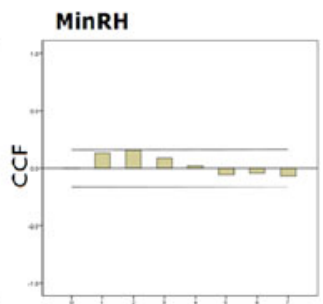

Lags (months)

Fig. 3. Plots of cross-correlation function (CCF) between monthly DF incidence rates separately and mosquito density, diurnal temperature range, timeliness of diagnosis and meteorological variation during January 2001 to December 2013 in Zhongshan, China. The two dashed lines illustrate critical values for cross-correlation (at the $5 \%$ level).

\section{DISCUSSION}

In this study, we explored the effect of potential risk factors such as timely diagnosis, mosquito density, and the meteorological factors on DF incidence rates in Zhongshan using time-series regression tree models. This study indicates that the timeliness of diagnosis, mosquito density, and temperature fluctuations played significant roles in the transmission of autochthonous DF in Zhongshan.

To some extent, the timeliness of diagnosis could reflect the sensitivity of the NIDRS run by the local CDC in China. The time interval between symptom onset and laboratory diagnosis contributed to the delay in reporting from the doctors to the surveillance system. The results of this study indicate that the early diagnosis of DF was the most important factor for reducing DF spread. We found that MTIOD was 6.044 days during the study period (Table 1). The threshold value of $4 \cdot 15$ days at a $1-2$ months lagged MA of MTIOD is the most important factor in determining the monthly incidence rates of the autochthonous DF in Zhongshan.

Previous studies suggest that timeliness of diagnosis is associated with patients' age, education, income, occupation, doctors' skills and diagnostic methods [30]. According to the current status of DF prevention and control strategies in China, several possible reasons related to the healthcare system and patients were considered as contributing factors to delayed diagnosis. First, the lack of specificity of clinical diagnosis and laboratory-confirmation facilities and health conditions of the basic community hospitals or primary healthcare departments can lead to the delay in diagnosis. Second, the social demography information of patients such as age, education, income, and occupation may impact on the timeliness for seeking the medical service. In our study, 59·14\% of all the reported cases were people aged 20-40 years. Patients were most commonly employed in the transport, production, and agricultural industries. A portion of suspected cases who presented with a history of sudden onset fever might take antifebrile medicine themselves, instead of consulting a doctor at the hospital during the acute febrile phase, leading to underestimation or delayed diagnosis. Additionally, DF cases are not quarantined, which could increase the opportunity for mosquito-human DENV transmission by human movement during the incubation period [31]. Therefore, this study indicates that any delay in diagnosis and notification of cases increases the risk of introducing the virus into mosquitoes and driving autochthonous transmission [3].

A number of changes need to be implemented to improve detection and to facilitate prevention and control in the community. First, clinical guidelines and management strategies for early monitoring and diagnosis of DF need to be improved. Professional education and training of the clinical doctors, nurses and other healthcare workers with respect to diagnosis of DF cases should be widely implemented. Second, 


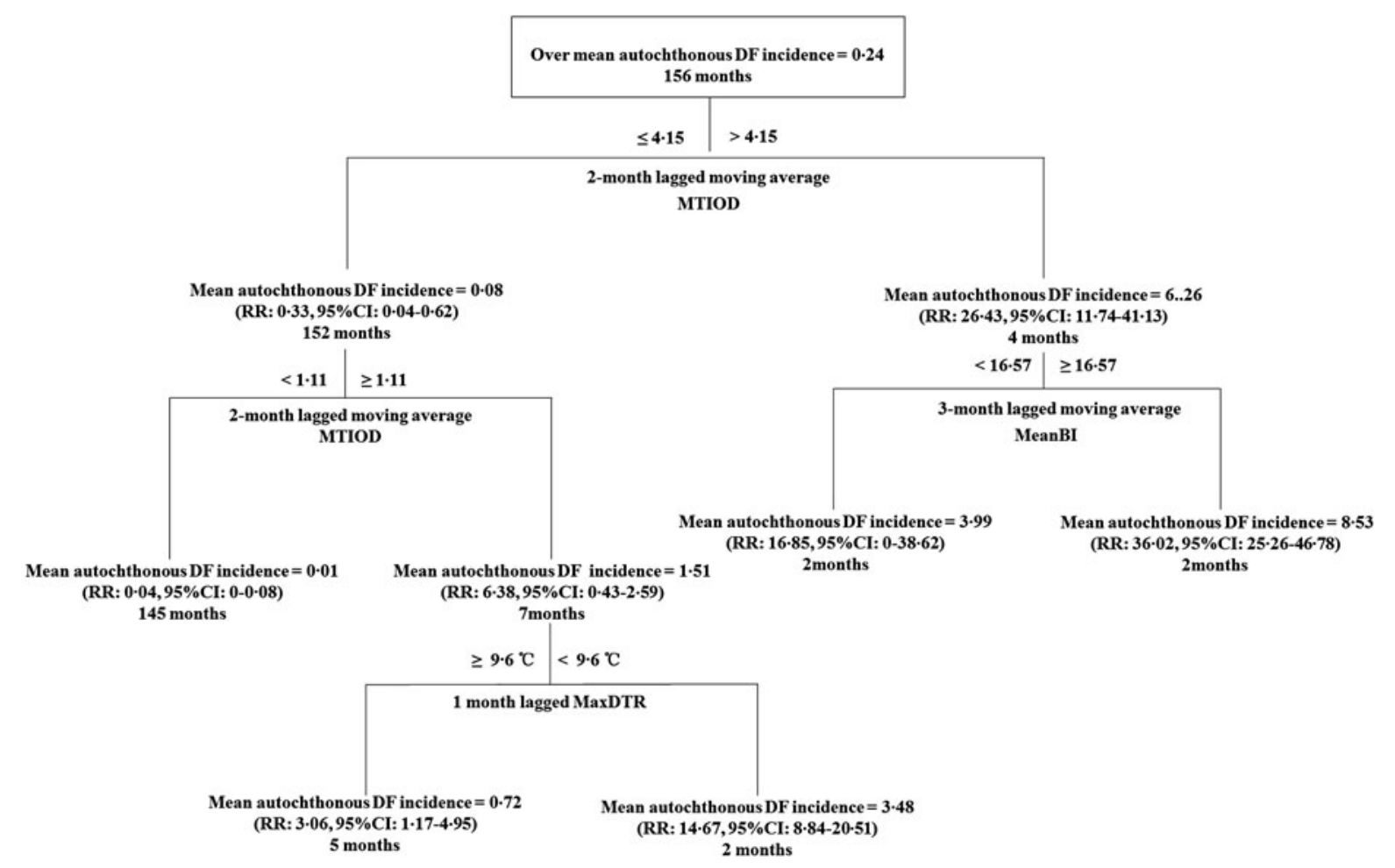

Fig. 4. Results of time-series classification and regression tree modelling the relationship between mosquito density, diurnal temperature range and the time interval between onset and diagnosis and monthly autochthonic DF incidence rates during the period January 2001 and December 2013 in Zhongshan, China. The regression tree shows the monthly mean autochthonic incidence rates of DF, the threshold values of the tree.

increased explorations of feasible and low-cost methods for early diagnosis are required for basic medical units. The NS1-based test as a rapid screening tool is utilized for the early diagnosis of DF, although the accuracy and variance between the primary and secondary infections of the NS1 test for early diagnosis of DF infection still needs to be enhanced in basic healthcare departments, the method's value remains [32]. Third, effective public health education for the population, especially for those at high risk needs improvement. Finally, customs officers should be involved in the prevention of epidemics; customs officers are often the first contact with imported cases.

Short-term temperature fluctuations were observed to be positively associated with autochthonic DF transmission in our study. These findings are consistent with previous studies $[11,33]$. Temperature is critical to the survival of the adult female Aedes mosquito and to virus transmission. Recent studies have explored the interaction between temperature fluctuations and mosquito-borne flavivirus transmission [13, 33]. A previous study found that larger diurnal temperature fluctuations could have a negative effect on vector survival for mosquitoes [12]. Moderate fluctuations in temperature $\left(10^{\circ} \mathrm{C}\right)$ would increase DF risk in the higher DF transmission season [13]. Temperature could influence the EIP of Aedes mosquitoes. Carrington et al. [34] showed that the large DTR might reduce DENV susceptibility and extend the EIP of mosquitoes. Our study indicates that when MaxDTR, at a lag of 1 month, was $<9 \cdot 6{ }^{\circ} \mathrm{C}$ and the 1-2 months lagged MA of MTIOD was $1 \cdot 11-4 \cdot 15$ days, the autochthonous DF incidence rate was $14 \cdot 67$-fold higher than the average autochthonous incidence rate of DF. The small DTR might be the key risk factor in DF transmission due to increased longevity, midgut infection rates and shortened EIP of Aedes mosquitoes compared to the large temperature fluctuations or constant temperature [34].

The main vector species for DF in China is A. albopictus [35]. Although DF transmission depends directly on female mosquitos, traditional larval indices such as BI are still widely used for the entomological surveillance during epidemic periods [36, 37]. In a 
study conducted in Vietnam, Pham et al. showed that there was a linkage between mosquito indices and DF incidence rate [38]. A Brazilian study identified that Aedes larval indices and rainfall could enhance DF outbreaks [39]. The time-lag effect between peak larval mosquito density and peak incidence rates of DF outbreaks was about 1 month [39]. We found the monthly autochthonous incidence rates of DF to be significantly positively correlated with the MeanBI at lags of 1-3 months (Fig. 3). Monthly autochthonous DF incidence rate increased by $36 \cdot 02$-fold when the 3-month lagged MA of the mean BI $\geqslant 16.57$ and the 2-month lagged MA of MTIOD was $>4 \cdot 15$ days (Fig. 4). BI, as a predictor to identify high-risk areas for DF transmission, was evaluated in Havana, Cuba [40]. This study showed BI $\geqslant 16.57$ to be a significant predictor. The threshold effects of A. albopictus density in our study were different from other studies [40, 41], which might be explained by ecological factors. Additionally, climate change, global travel, herd immunity, DENV serotypes, and timeliness of diagnosis would be possible factors to influence the relationship between the Aedes density and DF transmission risk [42, 43].

This study demonstrated the hierarchical interactions between predictors of DF using the time-series regression tree. It evaluated the relevance among the time interval between symptom onset and diagnosis, diurnal temperature fluctuations, mosquito density and autochthonic DF incidence rates in China using a time-series regression tree model construction. It suggests that the assessment of threshold effect of the important risk factors of DF and the implementation of management control measures could be attributed to DF transmission. Regression tree models can provide a non-parametric approach that can potentially better accommodate these complex interactions since they avoid some of the assumptions associated with linear regression [44].

In our previous study, we also compared the outcomes of the Bayesian CART model, with those of the traditional CART model. The analyses indicate that the nature and magnitude of the effect estimates were similar for the two methods [45]. Additionally, we tested the autoregression (AR) term in the CART model. The results showed that the key environmental factors remain significant in the relationship in the model even if we included the AR term in the model. However, the goodness-of-fit analyses indicate that the model without the AR term had higher accuracy than the model with the AR term [model with AR term: root mean squared error $(\mathrm{RMSE})=0 \cdot 528$; without AR term: RMSE $=0 \cdot 356]$. As the study aimed to assess and select the key environmental factors we did not include the AR term in the non-parametric model.

We suggest that local public healthcare departments should invest further in DF health education, enhance laboratory diagnostic services, focus more on Aedes mosquito density, and improve the sensitivity and specificity of surveillance systems, focused on both vectors and patients.

There were, however, a number of limitations to this study which need to be acknowledged. First, monthly data were used in the study. Weekly data would be better to assess the relationships between weather, mosquito density, and DF. Unfortunately, in this study, weekly data on mosquito density were unavailable. Second, the potential risk factors (herd immunity, human behaviour, virus strain and mosquito survival rate) were not included in the analysis as they were not available. It is suggested that the rapid and community-based diagnostic methods for decrease of DF infection and the spread in the mosquito-human interface are needed in future studies. Third, we did not evaluate the lifespan of larvae and the mosquito-human contact rate, thus the analysis was on the basis of a hypothesis that there was a positive correlation between mosquito density, biting rate, and infection rate. This study was an ecological study, which applied links between the risk factors and outcome of the diseases based on the population. Further research is needed to determine the possible causal association between the factors and DF incidence rates.

\section{CONCLUSION}

In this study, time-series regression tree models were used to assess and predict the risk of DF. We emphasize that timeliness of diagnosis, mosquito density, and diurnal temperature fluctuation play key roles in autochthonous DF transmission. MTIOD was determined to be the most critical factor in the assessment of the risk of DF transmission and the implementation of management control measures. Public health departments in China should pay more attention to mosquito control. The threshold effects of meteorological variation such as MaxDTR may be considered as a predictor of DF incidence rate. We conclud that the threshold effect of timeliness of diagnosis, mosquito density and diurnal temperature fluctuation 
examined in the time-series regression model could be used to predict and assess the risk of DF epidemics, and the factors identified is this study can benefit the early warning infectious diseases surveillance system.

\section{SUPPLEMENTARY MATERIAL}

For supplementary material accompanying this paper visit https://doi.org/10.1017/S095026881600265X.

\section{ACKNOWLEDGEMENTS}

We greatly acknowledge the contribution of $\mathrm{Mr}$ Yanheng $\mathrm{Wu}$, Ms. Wuyang Shi, Ms. Jinsi Lin and other clinical, laboratory of the Zhongshan CDC. The collaboration of data has benefited from the support of Mr Le Luo, Ms. Xueqin Chen who assisted in collecting data in Zhongshan, China. We thank Dr Gabriel J. Milinovich from the Queensland University of Technology, Australia for valuable comments on the manuscript. We also thank the Australia China Centre for Public Health (ACCPH) for supporting the project. Dr. Wenbiao Hu was supported by an Australian Research Council Future Fellowship (FT140101216).

This work was supported by the National Natural Science Foundation of China (NSFC) (grant no. 81373050, 81172735 International Program for $\mathrm{Ph}$. D. Candidates, Sun Yat-Sen University, China (grant no. 02300-18814201).

\section{DECLARATION OF INTEREST}

None.

\section{REFERENCES}

1. World Health Organization. Dengue (http://www.who. int/immunization/diseases/dengue/en/). Accessed 10 July 2015.

2. Guzman MG, Harris E. Dengue. Lancet 2015; 385: 453-465.

3. Simmons CP, et al. Current concepts. Dengue. New England Journal of Medicine 2012; 366: 1423-1432.

4. Jones KE, et al. Global trends in emerging infectious diseases. Nature 2008; 451: 990-993.

5. Schwartz E, et al. Seasonality, annual trends, and characteristics of dengue among ill returned travelers, 1997-2006. Emerging Infectious Diseases 2008; 14: 1081-1088.
6. Huang $\mathbf{X}$, et al. Imported dengue cases, weather variation and autochthonous dengue incidence in Cairns, Australia. PLoS ONE 2013; 8: e81887.

7. Banu S, et al. Dengue transmission in the Asia-Pacific region: impact of climate change and socio-environmental factors. Tropical Medicine \& International Health 2011; 16: 598-607.

8. Johansson MA, Dominici F, Glass GE. Local and global effects of climate on dengue transmission in Puerto Rico. PLoS Neglected Tropical Diseases 2009; 3: e382.

9. Horta MA, et al. Temporal relationship between environmental factors and the occurrence of dengue fever. International Journal of Environmental Health Research 2014; 24: 471-481.

10. Hu W, et al. Spatial patterns and socioecological drivers of dengue fever transmission in Queensland, Australia. Environmental Health Perspectives 2012; 120: 260-266.

11. Delatte $\mathbf{H}$, et al. Influence of temperature on immature development, survival, longevity,fecundity, and gonotrophic cycles of Aedes albopictus, vector of chikungunya and dengue in the Indian Ocean. Journal of Medical Entomology 2009; 46: 33-41.

12. Paaijmans KP, Read AF, Thomas MB. Understanding the link between malaria risk and climate. Proceedings of the National Academy of Sciences USA 2009; 106: 13844-13849.

13. Lambrechts L, et al. Impact of daily temperature fluctuations on dengue virus transmission by Aedes aegypti. Proceedings of the National Academy of Sciences USA 2011; 108: 7460-7465.

14. Fan WF, Yu SR, Cosgriff TM. The reemergence of dengue in China. Reviews of Infectious Diseases 1989; 11 (Suppl. 4): S847-853.

15. Lai S, et al. The changing epidemiology of dengue in China, 1990-2014: a descriptive analysis of 25 years of nationwide surveillance data. BMC Medicine 2015; 13: 100 .

16. Lambrechts L, Scott TW, Gubler DJ. Consequences of the expanding global distribution of Aedes albopictus for dengue virus transmission. PLoS Neglected Tropical Diseases 2010; 4: e646.

17. Tang KF, Ooi EE. Diagnosis of dengue: an update. Expert Review of Anti-infective Therapy 2012; 10: 895-907.

18. Bisset JA, et al. Insecticide resistance in two Aedes aegypti (Diptera: Culicidae) strains from Costa Rica. Journal of Medical Entomology 2013; 50: 352-361.

19. Zhongshan Statistical Bureau. Census data (http://www. zsstats.gov.cn). Accessed 10 July 2015.

20. National Health and Family Planning Commission of the People's Republic of China. Diagnostic criteria for dengue fever (WS 216-2008). (http://www.moh.gov.cn/ publicfiles/business/cmsresources/zwgkzt/cmsrsdocument/ doc3264.pdf). Accessed 10 July 2015.

21. National Health and Family Planning Commission of the People's Republic of China. Diagnostic criteria for dengue fever (WS 216-2001) (http://www.nhfpc.gov.cn/zwgkzt/ s9491/201212/34051.shtml). Accessed 10 July 2015. 
22. World Health Organization. Dengue: Guidelines for diagnosis, treatment, prevention and control (http://apps. who.int/iris/bitstream/10665/44188/1/9789241547871_eng. pdf). Accessed 10 July 2015.

23. World Health Organization. Dengue and severe dengue (http://www.who.int/mediacentre/factsheets/fs117/en/). Accessed 10 July 2015.

24. Zhongshan Meteorological Bureau. (http://www.zsqx. com/weather/). Accessed 10 July 2015.

25. Liu L, et al. Laboratory study on interspecific competition between Aedes albopictus and Aedes aegypti. Chinese Journal of Vector Biology and Control 2004; 15: 264-265.

26. Bowerman BL, O'Connell RT. Forecasting and Time Series: An Applied Approach, 3rd edn. Duxbury Press, 1993, pp. 726.

27. De'ath GFK. Classification and regression tree: a powerful yet simple technique for ecological data analysis. Ecology 2000; 81: 3178-3192.

28. Elith J, Leathwick JR, Hastie T. A working guide to boosted regression trees. Journal of Animal Ecology 2008; 77: 802-813.

29. Breiman L, et al. Classification and Regression Trees. Belmont, CA: Wadsworth International Group, 1984.

30. Yoo HS et al. Timeliness of national notifiable diseases surveillance system in Korea: a cross-sectional study. BMC Public Health 2009; 9: 93.

31. Stoddard ST, et al. House-to-house human movement drives dengue virus transmission. Proceedings of the National Academy of Sciences of the United States of America 2013; 110: 994-999.

32. Thomas SJ. NS1: a corner piece in the dengue pathogenesis puzzle? Science Translational Medicine 2015; 7: $304 f$ s37.

33. Paaijmans KP, et al. Influence of climate on malaria transmission depends on daily temperature variation. Proceedings of the National Academy of Sciences of the United States of America 2010; 107: 15135-15139.

34. Carrington LB, et al. Reduction of Aedes aegypti vector competence for dengue virus under large temperature fluctuations. The American Journal of Tropical Medicine and Hygiene 2013; 88: 689-697.

35. Wu F, et al. Distribution of Aedes albopictus (Diptera: Culicidae) in northwestern China. Vector Borne and Zoonotic Diseases (Larchmont, NY) 2011; 11: 11811186.

36. Tun-Lin W, et al. Critical examination of Aedes aegypti indices: correlations with abundance. The American Journal of Tropical Medicine and Hygiene 1996; 54: 543-547.

37. Focks DA. A Review of Entomological Sampling Methods and Indicators for Dengue Vectors. Geneva: World Health Organization, 2003.

38. Pham HV, et al. Ecological factors associated with dengue fever in a Central Highlands province, Vietnam. BMC Infectious Diseases 2011; 11: 172.

39. Pontes RJ, et al. Vector densities that potentiate dengue outbreaks in a Brazilian city. The American Journal of Tropical Medicine and Hygiene 2000; 62: 378-383.

40. Sanchez L, et al. Aedes aegypti larval indices and risk for dengue epidemics. Emerging Infectious Diseases 2006; 12: 800-806.

41. Focks DA, et al. Transmission thresholds for dengue in terms of Aedes aegypti pupae per person with discussion of their utility in source reduction efforts. American Journal of Tropical Medicine and Hygiene 2000; 62: 11-18.

42. Huang X, et al. El Nino-Southern Oscillation, local weather and occurrences of dengue virus serotypes. Scientific Reports 2015; 5: 16806.

43. van Panhuis WG, et al. Region-wide synchrony and traveling waves of dengue across eight countries in Southeast Asia. Proceedings of the National Academy of Sciences of the United States of America 2015; 112: 13069-13074.

44. De'ath G. Multivariate regression trees: a now technique for modeling species-environment relationships. Ecology 2002; 83 1105-1117.

45. Hu W, et al. Bayesian classification and regression trees for predicting incidence of cryptosporidiosis. PLoS ONE 2011; 6: e23903. 